\title{
Circuit
}

Musiques contemporaines

CIRCUIT

\section{Langage, musique et cognition : quelques remarques sur l'évolution nécessaire des problématiques psychologiques des vingt dernières années}

\section{Language, Music and Cognition: Some Remarks on the Necessary Evolution of Psychological Problematics of the Last Twenty Years}

\section{Michel Imberty}

Volume 13, numéro 2, 2003

Qui écoute? 1

URI : https://id.erudit.org/iderudit/902275ar

DOI : https://doi.org/10.7202/902275ar

Aller au sommaire du numéro

Éditeur(s)

Les Presses de l'Université de Montréal

ISSN

1183-1693 (imprimé)

1488-9692 (numérique)

Découvrir la revue

Citer cet article

Imberty, M. (2003). Langage, musique et cognition : quelques remarques sur l'évolution nécessaire des problématiques psychologiques des vingt dernières années. Circuit, 13(2), 93-110. https://doi.org/10.7202/902275ar
Résumé de l'article

L'application par Lerdahl et Jackendoff du modèle linguistique génératif de Chomsky à la musique tonale bute sur le postulat de l'innéité des compétences et pose le problème crucial de sa propre généralisation à toute musique. Le cognitivisme neurophysiologique propose des éléments de solution à travers le modèle de la modularité notamment, mais la dichotomie fodorienne entre périphérie et niveau central ne tient pas à l'observation des faits complexes. De manière générale, il convient de substituer à une ontologie des objets celle des événements.

L'auteur montre comment, dans ses propres travaux, il est parti d'une réactivation de la notion piagétienne de " grammaire d'enfants " pour proposer une "grammaire musicale évolutive »; ce modèle engendre des structures dynamiques, globales, orientées temporellement, qui sont antérieures aux notions d'intervalles de hauteur et de durée - déplaçant ainsi les apories du cognitivisme précédemment décrites et fournissant un substrat à toute approche de la musique intégrant prioritairement sa qualité temporelle. 


\title{
Langage, musique et cognition : quelques remarques sur l'évolution nécessaire des problématiques psychologiques des vingt dernières années
}

\author{
Michel Imberty
}

L'un des enjeux majeurs du cognitivisme dans les sciences humaines depuis vingt ans est sans doute de présenter une théorie cohérente et aussi générale que possible de l'ensemble des activités humaines par des systèmes de compétences plus ou moins spécifiques à tel ou tel domaine, mais dont les structures et les règles de fonctionnement sont identiques. En outre, ces compétences, qui sont donc particulières quant à leur contenu mais constituent un vaste ensemble cohérent de fonctions, sont innées'.

Il est facile de reconnaître dans ce credo ce qui furent les idées de départ de Chomsky, lorsqu'il proposa en 1957 la première formulation de sa fameuse grammaire générative. Depuis, la démarche chomskyenne a largement pénétré tous les domaines de la psychologie, d'abord le champ de la psycholinguistique, puis le champ de la psychologie de l'intelligence et de la pensée, aujourd'hui le champ de la psychologie de la musique. Or, les développements les plus récents ne sont pas sans conséquences sur notre conception de la musique aussi bien que sur notre conception du fonctionnement du cerveau humain en général, et je voudrais montrer comment certains des aspects les plus importants de ces théories doivent aujourd'hui être remis en cause si nous voulons comprendre réellement ce qu'est la cognition musicale en regard d'autres formes de cognition, que ce soit dans le domaine du langage ou dans celui de l'affectivité et des émotions.
1. Cet article est une version remaniée et résumée d'un texte paru en langue italienne dans la Rivista ltaliana di Musicologia, sous le titre «Le discipline musicologiche : prospettive di fine secolo», 2000, vol. XXXV, $n^{\text {os }} 7-2$, p. $411-452$.

\section{1 - Le modèle linguistique génératif appliqué à la musique}

C'est à partir d'une réflexion sur les travaux de deux chercheurs américains du début des années 1980 que j'aborderai ces questions. Lerdahl et Jackendoff ont 
en effet tenté d'appliquer à la musique (tonale) la démarche que Chomsky avait élaborée pour l'étude du langage. Ces travaux, comme ceux des linguistes générativistes, reposent sur des présupposés qui ne sont pas toujours explicités et qui pourtant orientent de manière décisive les conclusions qui peuvent être formulées, parce que ces présupposés dénotent une conception très particulière de la musique comme du langage, ce que l'on pourrait résumer ainsi :

1 - II existe des capacités, ou "compétences" spécifiques au langage d'une part, à la musique d'autre part, ces compétences pouvant être décrites dans leur fonctionnement sous forme de "grammaires", c'est-à-dire de systèmes capables de "générer" des séquences linguistiques ou musicales, indépendamment de tout apprentissage. En ce qui nous concerne, les compétences musicales constituent un ensemble original d'aptitudes ou de capacités innées dont le bon fonctionnement ne dépend que très peu des conditions particulières d'exercice concret au cours de l'enfance puis de la vie adulte. Est-ce le retour à une psychologie du «don» musical?

2 - Il existe des universaux linguistiques et musicaux caractéristiques de la pensée humaine; ceux-ci sont exprimés par des "règles de base" qui constituent une grammaire "noyau" commune à toutes les langues d'une part, commune à tous les systèmes musicaux d'autre part. Ces règles de base produisent les séquences types ou les "formes" que l'on retrouverait partout, dans toutes les cultures. En ce qui a trait à la musique, la confrontation des diverses grammaires musicales doit peu à peu permettre de mieux cerner ce que sont ces "formes élémentaires" universelles que l'on retrouverait dans toutes les cultures musicales, formes universelles qui ne devraient donc leur structure qu'aux systèmes psychologiques qui les ont produites et dont, par hypothèse, on pose qu'ils sont communs à tous les êtres humains.

3 - Les systèmes grammaticaux, dans la mesure où ils sont une formalisation des compétences psychologiques, doivent aussi trouver leur équivalent dans le fonctionnement interne du cerveau, ce qui signifie que les compétences correspondent à des systèmes neuronaux définis et indépendants les uns des autres. Pour la musique, là encore, diverses hypothèses ont été développées, dont celles portant sur les systèmes neuronaux modulaires.

L'une des hypothèses les plus fortes, et donc les plus contraignantes des théories génératives, que ce soit en linguistique ou en musique, est bien celle de l'innéité des compétences. Or, très rapidement, cette hypothèse peut perdre tout son sens en fonction de la façon dont on la précise ou non. L'un des arguments en faveur de cette innéité est que le langage, comme la musique, sont des activités spécifiquement humaines qu'on ne retrouve pas dans le monde animal. Mais lorsqu'on a dit cela, on n'a rien dit sur le degré de spécification des compétences correspondantes. Autrement dit, à quel degré de généralité peut-on décrire les compétences universelles? Dans le domaine du langage, il a été possible, jusqu'à un 
certain point, de montrer que les structures profondes reposent, dans presque tous les cas étudiés, sur des règles d'engendrement identiques et sur des fonctions identiques. Mais en musique, jusqu'à aujourd'hui, la seule tentative vraiment sérieuse pour définir des structures un tant soit peu générales et produites par des capacités innées reste celle de la Generative Theory of Tonal Music, proposée par Lerdahl et Jackendoff en 1983. Toutefois cette tentative se présente bien comme une théorie de la musique tonale, et il n'est guère possible de dire avec précision quelles en sont, de ce fait, les limitations. Il est très vraisemblable que ce qui est décrit dans cette théorie et que les auteurs appellent la structure des groupements a une valeur de généralisation qui va au-delà de la seule musique tonale occidentale. Mais il ne nous est guère possible de dire si, pour autant, ces phénomènes, dans leur généralité, renvoient à une compétence cognitive innée, car, contrairement à ce qui se passe pour le langage, nous ne pouvons pas falsifier les exemples engendrés par le modèle autrement que d'une manière peu contraignante et faible. Comment, par exemple, peuton définir une "bonne» séquence mélodique? Ou plus exactement, comment peuton la falsifier? Une ou plusieurs modifications n'entraîneront que des modifications de surface, et le sujet ne jugera pas la nouvelle séquence "mélodique" ou "non mélodique» (comme le locuteur jugera "grammaticale» ou "non grammaticale » une phrase de sa propre languel, mais seulement plus ou moins mélodique, plus ou moins surprenante, plus ou moins bien organisée. Si l'on pouvait définir la compétence musicale de la même manière que la compétence linguistique, le sujet serait alors capable de dire, entendant une séquence sonore, "c'est de la musique" ou "ce n'est pas de la musique". Mais un tel jugement ne peut être produit qu'en référence à un contexte culturel et historique déterminé, non par rapport à une universalité des structures de la musique en général et de la pensée musicale en général.

On saisit d'ailleurs là une différence profonde entre le langage et la musique : les grammaires musicales, que ce soit celle de Lerdahl et Jackendoff ou la théorie de l'Ursatz de Schenker dont elle s'inspire, procèdent de la surface vers la "base » par réductions successives qui, pour autant, n'en conservent pas moins quelque chose comme le "squelette" de la phrase, son expression tonale la plus simple, laquelle reste tout à fait correcte du point de vue du sens de la musique. Simplement, cette séquence simplifiée nous paraît banale, sans grand intérêt esthétique (voir les nombreux exemples donnés par les auteurs eux-mêmes). Au contraire, les réécritures successives dans la grammaire chomskyenne aboutissent à des structures de base qui ne sont pas des phrases et qui mettent en jeu des procédures d'engendrement des phrases en surface.

La difficulté pour circonscrire l'innéité de la compétence musicale apparaît donc double: 1) les grammaires musicales retrouvent - du moins n'ontelles pu jusqu'ici aller au-delà - au terme des réductions successives, des formes let non des fonctions) réputées élémentaires et simples, des schémas prototypiques qui ne peuvent donner lieu à des développements en surface que par extension et complexification. 
Toute la compétence musicale se réduirait alors à la capacité de produire des variations-développements, toute séquence pouvant apparaître comme une complexification ou une simplification d'une autre, sans limitation possible des récurrences. 2) L'innéité de la compétence musicale (en dehors des arguments philosophiques ou anthropologiques) est seulement induite à partir de l'universalité de ces formes simples, de ces schémas prototypiques, de ces patterns, ce qui signifie que ce ne sont pas les procédures d'engendrement qui sont premières (comme dans le langage où la phrase est générée à partir de fonctions syntagmatiques), mais bien ces groupements dits "naturels", dont il faut alors supposer qu'ils correspondent à des équilibres psychologiques ou physiologiques fondamentaux, eux-mêmes caractéristiques du fonctionnement mental. C'est, sous une autre forme, ce qu'exprimait déjà Rameau (Observations sur notre musique) en 1754 : "La musique nous est naturelle; nous ne devons qu'au pur instinct le sentiment qu'elle nous fait éprouver; ce même instinct agit en nous à l'occasion de plusieurs autres objets qui peuvent bien avoir quelque rapport avec la musique. »

\section{2 - Le cognitivisme neurophysiologique}

Il y a, dans toutes les théories génératives des compétences humaines, un raisonnement implicite qui pourrait devenir circulaire par dérive de psychologisme ou de cognitivisme. Dans le cas de la théorie générative de la musique tonale, ce cercle virtuel est le suivant : on postule l'existence d'une compétence musicale innée capable de permettre à l'auditeur de comprendre un nombre infini de phrases musicales. Comme le système tonal permet plus facilement que tout autre de construire un modèle fortement contraint, hiérarchisé et grammaticalisé /du fait de l'importance des fonctions au sein du système), on construit donc un modèle de cette compétence à partir de l'analyse du système tonal, et notamment à partir des procédures réductrices de l'analyse. On arrive alors à l'idée que l'auditeur, pour comprendre une phrase de musique (tonale) la réduit, c'està-dire la simplifie en recherchant les éléments les plus importants de la structure et en construisant un schéma économique fortement hiérarchisé. Les procédures de simplification et de réduction sont bien directement formalisées à partir des propriétés internes du système tonal. On fait alors l'hypothèse que ces opérations de simplification et de réduction sont aussi des opérations mentales (pas seulement des procédures d'analyse) et le modèle apparaît alors comme celui de la compétence (psychologique) musicale biologiquement déterminée dont on avait fait l'hypothèse au départ. Rien n'est d'ailleurs plus facile que de prouver la réalité psychologique de cette grammaire tonale : il suffit de travailler, selon des paradigmes expérimentaux désormais classiques en linguistique, sur de la musique tonale, ce que font la plupart des travaux en vogue aujourd'hui. 
Pour éviter de s'enfermer dans ce cercle cognitiviste caractéristique, il n'y a que deux issues possibles : soit prouver non plus seulement la réalité psychologique de la théorie générative de la musique tonale ( $c$ 'est-à-dire la réalité psychologique des procédures de réduction), mais la réalité et la spécificité des circuits neuronaux correspondant à cette compétence; soit chercher à vérifier certaines propriétés des formes tonales (structures de regroupement, structures métriques, voire certaines structures de prolongations) dans la compréhension de la musique atonale. Dans I'un et l'autre cas, l'entreprise est difficile et l'issue, encore aujourd'hui incertaine.

On peut illustrer le genre d'hypothèses et de recherches auxquelles donne lieu la première direction à travers le modèle connu sous le nom de modèle de la modularité : selon ce modèle, le système cognitif humain est composé de sous-systèmes physiquement séparés qui correspondent chacun à un corpus spécifique de connaissances et de procédures. Ces sous-systèmes sont autonomes et peuvent être modifiés sans que l'ensemble du système ne subisse de changements importants. Un tel système modulaire est plus économique et efficace que les systèmes non modulaires interdépendants, et il correspond assez bien à l'hypothèse générale du cognitivisme, à savoir l'existence de compétences spécifiques biologiquement déterminées et relativement indépendantes les unes des autres. Tant dans le domaine pathologique que dans le domaine de l'observation des faits de perception et de langage, il semble bien que l'on puisse trouver confirmation de l'hypothèse modulaire. C'est Fodor qui, dès 1983, détailla les diverses propriétés d'un système modulaire : les plus importantes sont 1) que les modules sont spécifiques à un champ d'activités et sont associés à des systèmes neuronaux spécifiques, identifiables, et qu'ils peuvent être perturbés ou détruits de manière spécifique par une lésion cérébrale ; 2) que les modules ont leur propre capacité de traitement et leurs propres ressources de mémoire et ils sont indépendants des autres modules et des processus plus généraux; 3) que l'action ou l' "opération" d'un module est très rapide, automatique et fonctionnelle, qu'elle correspond à une architecture neuronale fixe (circuit) ; 4) que l'intégration des connaissances fixées dans les modules est assurée par des processus centraux qui agissent à la sortie des modules, mais non à l'intérieur qui reste inaccessible.

L'existence de systèmes modulaires pour la perception et la compréhension de la musique a été également proposée par Gardner (1983) et Jackendoff (1987). Mais selon Fodor, il existerait des mécanismes modulaires distincts et très spécifiques pour le traitement des hauteurs (mélodie), et pour le traitement de l'organisation temporelle (rythme). Pourtant, la réalité est beaucoup plus complexe : selon divers travaux présentés par I. Peretz et J. Morais (1989), tout dépend du niveau de traitement de l'information musicale. Aux niveaux les plus précoces, le traitement des hauteurs et des durées est distinct, mais aux niveaux ultérieurs, il dépend des mécanismes centraux de coordination. On peut donc s'interroger sur la pertinence de l'hypothèse modulaire dès qu'on aborde les niveaux élaborés des séquences musicales, de leur compréhension et de leur mémorisation, puisqu'en 
fait, dans la plupart des systèmes musicaux, il y a dépendance entre les structures de hauteurs et les structures de durées. À moins de supposer que la tonalité en

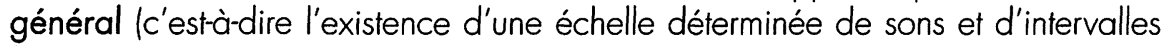
fixes) puisse se définir elle-même comme un système modulaire.

En réalité, le fonctionnement modulaire tel qu'il est décrit par Fodor ne touche que certains aspects de la musique, et probablement les moins élaborés et les moins... musicaux, des sons isolés ou des séquences de quelques sons! Par exemple, la spécialisation hémisphérique, hypothèse à partir de laquelle ont été élaborés les modèles modulaires, est probablement moins nette pour la musique que pour la parole. Ainsi, dès 1974, Bever et Chiarello avaient montré que musiciens et non musiciens traitaient les mélodies dans les hémisphères opposés. L'idée selon laquelle la perception et la compréhension de la musique sont basées sur des mécanismes très généraux non liés à des circuits neuronaux très délimités reste donc une idée tout à fait plausible, dès lors qu'on s'intéresse à l'intégration perceptive et mémorielle d'une phrase musicale d'une certaine durée, à plus forte raison d'une pièce tout entière. Mais cela détruit en grande partie l'idée de compétence musicale au sens des théories génératives d'inspiration chomskyenne, à moins de considérer que les grammaires sont, elles aussi, des systèmes modulaires. Et il est vrai que Lerdahl et Jackendoff présentent leur théorie en partie aussi comme un système modulaire, du moins comme la modélisation possible d'un système dont existerait le correspondant dans les circuits neuronaux du cerveau.

Or, rien ne permet de l'affirmer, non seulement parce que la preuve de l'existence de tels circuits dans le cerveau humain n'est pas faite, mais parce qu'en outre, la modélisation reste fermée sur la musique tonale, et qu'il existe bien d'autres musiques dont l'homme est capable de saisir les structures et les qualités esthétiques, ce qui est bien l'essentiel. On pourrait donc dire que ce qui reste ici inexpliqué par la modularité, c'est précisément le fonctionnement des systèmes centraux dont sans doute relève l'ensemble des activités musicales de niveau supérieur. La dichotomie fodorienne entre périphérie et niveau central (modules et processus centraux) ne tient pas à l'observation des faits complexes : comme l'écrit G. Tiberghien (1999), "tout converge, au contraire, vers l'hypothèse plus complexe d'un continuum de traitements, organisés selon une architecture hautement interactive et très probablement en cascade, possédant, à des degrés divers et sous des contraintes plus ou moins fortes, tel ou tel sous-ensemble de propriétés modulaires". C'est dans ces conditions que doit être abordé l'ensemble des processus de traitement de la musique par le cerveau humain, dans le contexte de son histoire et de ses cultures : celles-ci comme celle-là ne peuvent être prises en compte que dans une théorie beaucoup plus générale et peutêtre "hybride" des interactions systémiques, non seulement entre niveaux périphériques et niveaux centraux de traitement, mais entre systèmes endogènes et systèmes exogènes, c'està-dire entre systèmes internes à l'individu et systèmes du milieu biosocial. On ne serait pas loin de penser que l'ensemble de ces systèmes sont d'abord et avant 
tout soumis à des lois d'autorégulation qui leur permettent à la fois de s'équilibrer mutuellement, et aussi de faire surgir de nouvelles formes d'interactions processuelles porteuses de développement et de création : les crises, les révolutions, aussi bien dans le domaine des cultures que de l'histoire, doivent pouvoir être interprétées en termes d'interactions systémiques dynamiques, où les principes hiérarchiques de l'organisation tonale de la musique, entre autres, n'apparaissent plus comme inéluctables au nom des propriétés modulaires et à l'encontre de l'histoire. À ce niveau de généralité, rien n'exclut, selon moi, l'idée d'équilibration majorante chère à Piaget : l'ordre biologique est créateur par l'interaction avec le milieu, et les processus centraux du cerveau humain sont partie prenante directe de cette création : sans quoi, comment pourrions-nous comprendre la rupture que représente, dans notre histoire, la musique sérielle et avec elle tant d'autres courants du XXe siècle occidental?

\section{3 - La temporalité musicale, impasse épistémologique du cognitivisme}

On me permettra de revenir encore un instant à Piaget avant de prolonger les réflexions précédentes à propos du développement musical de l'enfant. On sait que pour Piaget, les concepts de réversibilité et de conservation (ou d'invariant) sont essentiels dans la théorie des opérations mentales. Cependant, dans la plupart des recherches qui s'inspirent de l'œuvre piagétien, on peut constater que ce sont les aspects statiques communs aux structures musicales et aux formes visuelles ou aux objets abstraits de la pensée qui ont d'abord retenu l'attention des chercheurs. Dans des travaux d'inspiration néo-piagétienne, notamment américains, on trouve des tentatives pour définir, à l'image des fameux invariants que sont l'objet permanent, la conservation de la quantité, du poids et du volume dans les expériences sur les transformations d'une boulette d'argile, des "invariants musicaux" comme le mètre, l'intervalle, la pulsation, etc. dont on montre qu'ils sont dépendants, dans leur construction, du développement global de la pensée opératoire.

Si j'ai moi-même adopté la perspective piagétienne au début de ma carrière de chercheur, notamment dans mon ouvrage de 1969, je n'y ai cependant pas abordé le problème de la construction de la pensée musicale chez l'enfant sous cet angle de l'invariance, mais plutôt d'un point de vue à la fois inverse et complémentaire : comment peut se construire un système de représentations mentales (musicales) sans réversibilité, et donc sans construction d'invariants au sens de la théorie des opérations? L'originalité de la perception de la forme musicale comme de toute forme temporelle - est bien qu'elle se déroule dans le temps, de manière irréversible. Le temps vécu, celui de la perception d'une mélodie par 
exemple, n'est pas le temps abstrait sur lequel on peut effectuer des opérations, la seule temporalité prise en compte par Piaget. La forme mélodique implique l'irréversibilité, et cela n'a rien à voir avec des symétries que l'on peut observer très facilement même dans les productions musicales les plus élémentaires. Certes, le système tonal suppose au contraire la réversibilité syntaxique dans le parcours tonique-dominante-tonique. Mais pour qu'elle soit perçue et ressentie comme nécessaire, il faut que le temps musical concret dans son déroulement soit mis entre parenthèses et qu'une structure abstraite soit mémorisée. Comment ce temps mémorisé, abstrait, "réversible" se construitil ? Autrement dit, comment se construit l'invariance non d'un élément (mètre, intervalle, pattern mélodico-rythmique...) mais d'un parcours dans le temps, comment se construit une boucle qui part d'un point pour revenir à ce point malgré l'irréversibilité temporelle? Ce que montrent en tout cas les résultats expérimentaux, c'est que le parcours syntaxique de base déterminé par l'enchaînement tonique-dominante-tonique et ses variantes n'est pas ressenti comme nécessaire psychologiquement avant l'âge de 10 ans, c'està-dire avant le début des opérations formelles qui permettent aussi les constructions temporelles abstraites. En réalité, le modèle piagétien ne permet pas de comprendre la nature des phénomènes qui caractérisent la perception musicale de l'enfant avant cet âge : la construction des structures culturellement tonales renforce les recentrations perceptives au détriment de décentrations qui ne s'opèrent que très lentement ensuite et sous l'action de l'apprentissage et de l'éducation. La fluidité et la temporalité sont éliminées des faits expérimentaux au profit des seules formes structurales conçues comme $n$ 'importe quelle structure linguistique ou spatiale. On retrouve là, à l'évidence, la primauté du discontinu sur le continu, c'est-à-dire la même limitation théorique que celle du cognitivisme récent.

En réalité, nous touchons à l'un des problèmes essentiels de la philosophie et de la psychologie de la musique aujourd'hui : comme le note J. Molino dans un article fort éclairant (1998), la difficulté majeure tient à ce que la tradition philosophique occidentale depuis l'âge classique est basée sur une ontologie des objets et non sur une ontologie des événements. Ce qui caractérise l'ontologie des objets est qu'elle formalise le discontinu, la délimitation spatiale, la séparation et la stabilité des limites. Les objets sont là, devant moi, et je peux alors les toucher, les regarder, les nommer. II n'en va pas de même des sons qui, par nature, apparaissent et disparaissent, et que je reconnais seulement comme les produits de sources que je cherche à identifier. Les sons ne sont pas des substances, ils sont des événements, et le discret, en musique, n'est que l'artefact de la pensée humaine qui sépare ef analyse sur fond de réalités objectales et non de réalités événementielles. Les sons ne sont pas des objets mais des processus et seule la volonté d'analyser l'expérience musicale en dehors de tout contexte subjectif et humain en fait une suite de notes : "La musique, écritil, ce n'est pas seulement du sonore, c'est du sonore transformé par l'expérience humaine... La musique ne se fonde pas sur des notes, des échelles ou des règles de succession harmonique mais sur des schèmes qui mêlent au sonore le perceptif, le moteur, l'affectif» (1998, p. 264). En reprenant sur ce 
point L. Meyer (1973), Molino ajoute : la mélodie, "en même temps que contour, est dynamisme, force orientée qui échappe par définition aux méthodes traditionnelles d'analyse» (1998, p. 265). C'est bien cette distinction objet/événement qui permet à J. Molino de réintroduire la temporalité dans la réalité physique de la musique comme dans sa réalité psychologique et sociologique. L'événement, c'est du temps, et l'espace n'en est que le réceptacle amorphe. L'événement se produit, il est rupture et lien à la fois : rupture parce qu'il renvoie au passé ce qui le précède (les événements antérieurs), lien parce qu'il permet d'articuler ce passé au futur qu'il ouvre dans ses conséquences prévisibles ou non ${ }^{2}$.

Au début des années 1980, le concept de "grammaires d'enfants" est largement utilisé en psycholinguistique sous l'influence des travaux de Chomsky. Je fus le premier à le transposer dans le domaine de la musique (Imberty, 1981, 1991). L'idée est que, si l'on veut comprendre la nature des processus cognitifs qui se construisent durant l'enfance, il faut d'une part s'intéresser autant à la production musicale qu'à la perception et à la mémorisation (reproduction, imitation, mais surtout improvisations dans des conditions variées), et qu'il faut d'autre part réintroduire la dimension développementale dans les recherches de systèmes de règles syntaxiques des productions. On a vu qu'à cette époque, il y avait un fort credo en faveur de l'existence de compétences spécialisées et innées, et dans cette perspective, les activités musicales, en particulier la production spontanée de séquences chantées ou instrumentalement jouées, pourraient être expliquées par un système de règles d'engendrement (une grammaire) décrivant à la fois les corpus concrets et les mécanismes cognitifs sous-jacents sans aucune référence à une "construction", une "maturation" ou un "développement». En outre, cette idée de compétence musicale va de pair avec la recherche d'universaux musicaux transcendant tous les systèmes particuliers et toutes les cultures, universaux qui seraient, comme dans tous les domaines des activités humaines, témoins de la nature biologique des compétences.

Si ce générativisme est intéressant parce qu'il permet d'aborder enfin les productions musicales des enfants de manière directe et non à partir des conduites perceptives, il n'en reste pas moins marqué de toutes les limitations dénoncées ci-dessus : il s'agit en gros de savoir comment se produisent des structures et non comment l'enfant, voire l'adulte, produit de la musique qui peut avoir son originalité propre, qui possède son dynamisme plus essentiel que les combinaisons de sons ef de durées décrites par les règles des grammaires ainsi créées par le chercheur. Ce qui manque encore ici, comme précédemment, c'est la prise en compte du temps et du mouvement, c'est la compréhension du lien entre ce mouvement et l'énergie (physique, sensorimotrice, psychologique...) mobilisée par le sujet dans son acte. C'est pourquoi j'élabore un modèle pour la construction de grammaires musicales évolutives dont les règles engendrent des séquences mélodico-rythmiques simulant les productions spontanées des enfants. Ces simulations sont ensuite confrontées à un corpus et validées. Ce concept de "grammaire évolutive" implique que les règles, dont on fait l'hypothèse qu'elles correspondent à des opérations mentales du
2. La matérialité de l'événement donne souvent l'illusion qu'il peut être circonscrit dans un lieu ou un espace déterminé, mais cette réalité-là n'est que secondaire par rapport à sa nature temporelle. L'événement suscite le mouvement, engendre le dynamisme inhérent à tout changement. Comme l'écrit J.M. Chouvel: "Avec l'irréversibilité, le temps apparaît essentiellement dissymétrique En ce sens, il est le contraire de ce que l'espace propose de plus remarquable [...]. Le Temps a un sens, et il convient de restituer intégralement ce sens dans toute notre démarche analytique, dans l'effort de notre réflexion sur la réflexion même» 11998 , p. 27). 
sujet, comportent des parties "obligatoires", fixes, décrivant des processus généraux stables, et des parties "non obligatoires" qui, elles, décrivent des processus d'évolution structurale prévisibles au cours du développement psychologique de l'enfant. Autrement dit, pour simplifier, les parties "obligatoires" des règles décriraient les processus constants, les opérations caractérisant le noyau fixe - peutêtre inné ? - de la compétence musicale, les parties «non obligatoires» décriraient au contraire la programmation des changements, des évolutions structurales conduisant à la forme finale de la compétence.

Mais ce qui est le plus remarquable, et qui vient fortement relativiser les apports du cognitivisme, est qu'aussi bien au niveau du noyau fixe qu'à celui des évolutions et des constructions au cours du développement, ce modèle engendre des structures dynamiques, globales, orientées temporellement, antérieures aux notions d'intervalles de hauteur et de durée. En effet, ce que j'ai pu montrer à partir de l'étude de corpus divers de productions d'enfants âgés de 5 à 10 ans (Imberty, 1981a, 1983), est que toute séquence vocale élémentaire produite spontanément comprend un élément permanent et stable, défini et discontinu, le pivot (intervalle de hauteurs ou intervalle de temps ou les deux), et un élément instable, flou, qui ne peut se caractériser autrement que comme un "remplissement» ou un "colmatage " de l'intervalle stable, élément par conséquent continu.

Sur cette structure alternée très simple se greffe un schème psychologique temporel : l'enchaînement d'un appui, suivi d'un élan qui crée la tension, et d'une chute qui clôt la période ou le segment. Tous les musiciens reconnaissent volontiers dans cette succession d'origine sensorimotrice le principe de formation d'une «figure musicale bien formée», selon l'expression de C. Deliège (1984, p. 177), ou encore cet enchaînement caractéristique qui définit une trame temporelle dans la grammaire générative de la musique tonale mise au point par Lerdahl ef Jackendoff : début structural, processus, conclusion structurale. Mais cette figure à trois places peut en fait se ramener à la simple alternance tension/détente, puisque l'appui initial n'est appui que dans la mesure où il permet à la tension de s'instaurer, au mouvement de s'élancer, si bien que l'appui n'a de sens que par rapport à la chute qu'il fait attendre. La figure musicale psychologiquement première est donc en réalité une figure dynamique, à deux places, un mouvement à deux temps : ici, un appui vocal qui lance une tension neuromusculaire pharyngée et respiratoire produisant une "séquence" indéterminée de sons de hauteurs et de durées variables, suivie d'une détente, d'un repos sur une finale stabilisée. Entre l'appui initial et l'appui final, il n'y a donc qu'une tension, une énergie qui doit se résoudre. Tout le dynamisme d'une progression musicale est déjà là, jeu d'équilibration entre un pivot stable (intervalle de hauteurs ou de durées) et un remplissage instable : on le rencontre très tôt, dans les premières activités sensorimotrices et les premières productions vocales du bébé, et c'est sans doute là une donnée universelle.

Cet enchaînement d'un appui, d'un élan et d'une chute constitue aussi le premier schème temporel orienté de la pensée musicale, et il se manifeste notamment dans 
la pratique du chant, au-delà sans doute dans toutes les activités vocales. Chercher en soi ce mouvement qui lance la voix dans son déploiement extérieur, prendre son souffle, c'està-dire prendre appui sur le corps entier dans une inspiration costoabdominale pour déployer le son dans la plénitude d'un legato parfaitement maîtrisé et pourtant libéré, c'est le premier apprentissage du chanteur. Or, plus cette inspiration initiale sera profonde, plus la mobilisation de l'énergie corporelle musculaire et nerveuse sera intense, plus l'expiration et la détente qui suivront apporteront le sentiment d'un aboutissement temporel serein conduit progressivement et sans heurt à son épuisement final. Appui sur toute l'énergie concentrée du corps, élan respiratoire, relâchement neuromusculaire dans l'expiration finale, telle est cette mise en ordre primitive qui commande toute succession sonore et la dirige, la tend vers ce point de non-retour où l'élan peut soit se renouveler, soit clore au contraire la séquence tout entière, avant même qu'une syntaxe ou un code ne formalise cet aboutissement et le fige dans une temporalité spatialisée et géométrisée ${ }^{3}$.

Je ne reviendrai pas en détail sur les règles de la grammaire évolutive que j'ai proposée. Je voudrais seulement souligner que ces règles sont des règles d'organisation des événements sonores dans le temps, dans les successions concrètes créées ou reproduites par les enfants. Là encore, grammaire de processus et non grammaire de structures d'objets ou d'éléments. D'où l'importance de cette distinction, sur laquelle repose le modèle, entre schèmes d'ordre et schèmes de relation $d^{\prime}$ 'ordre. Les schèmes d'ordre constituent l'ensemble des intuitions que le sujet a des successions temporelles sans qu'il ait conscience des éléments constitutifs de ces successions. Il s'agit donc d'intuitions de nature sensorimotrices ou représentationnelles dont les contenus sont indissociables des séquences ordonnées elles-mêmes. Par exemple, un enfant de 5 ans est capable de reproduire une mélodie même assez longue intégralement; mais s'il se trompe, il ne peut reprendre là où il s'est arrêté, la séquence n'ayant d'existence que globalement, et il doit reprendre du début. De même, il n'y a pas d'événements temporels privilégiés, par exemple pas de début et de fin structurés. Dans une séquence tonale qu'il n'a jamais entendue, si l'on s'arrête, pour lui, c'est que la séquence est finie. L'événement final n'est événement final qu' " après coup", il n'est pas celui qui vient après tous les autres, il est seulement dans le prolongement temporel de la séquence entière. En un mot, les schèmes d'ordre sont des schèmes de la continuité. Au contraire, les schèmes de relation d'ordre organisent la logique de la succession dans un temps contenant indépendant des événements contenus. Chaque événement, chaque note ou accord par exemple, voit sa place définie par rapport à l'ensemble des autres événements, c'està-dire par une syntaxe. Ce sont ces schèmes qui permettent notamment au sujet de maîtriser la construction formelle temporelle au-delà du temps concret dans lequel elle se déroule : repérer les thèmes, les motifs, les ressemblances et dissemblances à distance, bref, tisser des relations logiques entre les constituants de la forme dans un temps abstrait, un temps proprement délimité par la forme, un temps/espace de déploiement des structures. Les schèmes de relation d'ordre sont des schèmes de la discontinuité fonctionnelle et syntaxique. Quant
3. Ce même schème d'ordre ne préside-til pas aussi à toute exécution instrumentale? Ainsi, les professeurs de piano ne recommandent-ils pas à l'élève de "prendre sa respiration "avant de commencer?

Recommandation qui exprime précisément la nécessité de l'appui mental et corporel initial pour déployer ensuite la phrase à exécuter dans un élan qui en assure la continuité jusqu'à sa conclusion. Quel chef d'orchestre n'en a pas fait l'expérience avec l'attaque, par exemple, du final de la $39^{\circ}$ Symphonie de Mozart! Si aucun appui intériorisé n'est pensé par les musiciens avant de jover la tête du motif initial / $c$ 'est-à-dire si le troisième temps virtuel qui précède les quatre doubles croches initiales n'est pas mentalement exécutél, l'élan du thème en est complètement brisé et le jeu des tensions et détentes, anéanti. II y a là un phénomène très prégnant, une sorte de "pré-ordre » psychologiquement incontournable qui ne se manifeste pas en termes de structures mais d'abord en termes de processus orienté. 
aux schèmes d'ordre articulés, ils correspondent à l'identification encore tout intuitive d'événements privilégiés dans la succession temporelle et qui en scandent le déroulement, comme par exemple l'identification de formules initiales ou finales, de pivots particuliers, etc. Mais ces événements n'ont encore que des fonctions présyntaxiques, ils restent indissociables de la séquence (mélodie, par exemple) dont ils constituent la charpente perceptive. Le développement musical de l'enfant dans le monde occidental peut donc se décrire comme la construction progressive des schèmes de relation d'ordre, c'est-à-dire des schèmes qui permettent notamment d'organiser et de comprendre la musique tonale.

Mais cette construction n'annihile pas les schèmes d'ordre. Ceux-ci correspondent en fait à des capacités temporelles très générales mises en jeu dans la musique de toutes les cultures et de toutes les époques. Les phénomènes sensorimoteurs et dynamiques dont je viens de donner des exemples à propos du chant se retrouvent à la base de très nombreuses pratiques musicales et de très nombreuses formes de musique dans le monde. D'ailleurs, les premiers témoignages que nous avons de I'homo musicus sont des témoignages où la musique est totalement ancrée dans le corps et le souffle (battements de pieds et de mains, mouvements de danse, chants ou cris, souffles dans des trompes, des tuyaux de toutes sortes...). Dans tous les cas, le mouvement prime, l'acte prime, puis le sonore disparaît dans le temps et ne se reprend vie qu'avec le mouvement suivant: l'écriture n'existant pas, une telle musique est totalement dépendante et liée à celui qui, dans l'instant, la produit, et elle n'offre aucune prise à une abstraction de structures pré-établies, s'en trouvant d'ailleurs délaissée par l'analyse savante ${ }^{4}$.
4. J. P. Mialaret, dans un travail original sur les explorations instrumentales des enfants (1997), a fortement montré le rôle de ces vecteurs dynamiques dans la création et le développement des improvisations.

L. Miroudot (2000) reprend celte idée et montre comment les structures temporelles de durées, d'intensité, d'accents prévalent, chez les enfants de 5 ans, sur les structures de hau teurs. Non qu'on ne constate pas l'utilisation d'intervalles mélodiques de hauteurs précises, mais lorsqu'il s'agit bien de productions spontanées, et non d'imitations de modèles de l'adulte, c'est l'énergie déployée par le sujet pour extérioriser son geste vocal (Miroudot) ou instrumental (Mialaret) qui détermine la forme des intervalles, leurs arrangements en concomitance avec les durées, les attaques, les accentuations, les variations d'intensité. J'insiste sur le fait que c'est là le sens du processus de colmatage : il s'agit bien de remplir un temps, de dessiner un mouvement qui occupe ce temps engendré par l'élan initial. Le colmatage est un processus temporel, une énergie du corps qui passe dans la voix du jeune chanteur ou dans le bras et la main du petit instrumentiste. Le colmatage indique bien qu'il y a une direction, un but, puisqu'il s'agit de combler pour atteindre au but final, l'arrivée du geste musical lui-même.

\section{4 - Cognitivisme et psychologie des systèmes dynamiques}

Comme on l'a vu, il y a ancrage de la production musicale spontanée des enfants dans l'énergie corporelle. Mais comment se manifeste cette énergie? Jusqu'où cette induction des conduites musicales par le corps vattelle? Répondre à ces questions, c'est renover tout simplement avec toute une tradition de la psychologie développementale qui trouve l'origine de l'émotion et de la vie affective dans la tonicité et la motricité expressives. Mais c'est aussi se rappeler que, bien avant les formes très élaborées et abstraites de la communication sociale verbale, il existait des formes de communication sociale dans lesquelles le corps, les gestes, les attitudes, les mimiques, les jeux d'échopraxies autant que d'écholalies avaient un rôle décisif pour la maturation intellectuelle, sociale, affective ${ }^{5}$.

Ce que nous savons aujourd'hui du développement du nourrisson montre que celui-ci est plongé avant tout dans un univers de sons et de mouvements, avant
5. Un récent numéro spécial de la revue Musicæ Scientiæ (Rhythm, Musical Narrative, and Origins of Human Communication dans Musicæ Scientiæ, 1999-2000, $n^{\circ}$ spécial, 215 p.) a été consacré à ces questions : les diverses études présentées établissent clairement que la qualité et l'efficacité de la communication chez les tout petits enfants, entre eux et avec leur entourage adulte, dépend de ce qu'on peut bien appeler la «musicalité » des comportements interactifs. 
même d'être sollicité par les formes visuelles et les couleurs. Mais au-delà de ce bain sonore temporel, ce qui caractérise les premiers mois de la vie, c'est la naissance des rapports humains interactifs, des premiers rapports sociaux dans des comportements suscités par l'adulte (la mère le plus souvent) mais repris par l'enfant de manière active et créatrice, de telle sorte que se construisent des expériences à deux où l'échange de sensations, de rythmes et d'affects devient le moteur de la communication. Tout le début de la socialisation de l'enfant est basé sur une organisation répétitive créée par la mère dans ses relations avec lui. En fait elle utilise tous les registres comportementaux sur le mode de la répétition pour entrer en relation avec son enfant : vocalisations, mouvements, stimulations tactiles et kinesthésiques..., sans qu'il y ait, au moins au début, intention pédagogique. À propos des jeux vocaux de la mère et de son enfant, D. Stern écrit : "Ce qui sans doute importe moins, c'est ce que la mère dit réellement. L'important, c'est la musicalité des sons qu'elle produit. De ce point de vue, l'action répétitive acquiert son importance en tant qu'unité structurelle et fonctionnelle dans l'interaction " 11977 , p. 122). Et la structuration temporelle des interactions, ou des comportements interactifs, qu'engendrent les répétitions est sans doute le phénomène essentiel sur lequel doit s'appuyer notre compréhension du développement de la cognition musicale : cela a été bien montré par J. P. Mialaret (1997) dans sa description des conduites exploratoires instrumentales. D'ailleurs, les analyses de D. Stern ne peuvent ici que satisfaire le musicien : ce principe de répétition / variation repose sur deux éléments indispensables au développement de la socialisation, de l'affect et de la cognition. D'une part, l'enfant apprend à s'adapter à un nombre toujours plus important de variations, à la condition qu'elles ne s'éloignent pas trop du modèle initial, mais d'autre part, il ne le peut que parce que la répétition est basée sur un rythme régulier qui rend prévisible et organise le temps. C'est sur cette régularité que se fonde l'alternance émotionnelle de la tension et de la détente, de l'insatisfaction et de la satisfaction, en même temps que de leurs diverses transpositions, de leurs divers contournements. En bref, on voit que tout le développement du comportement social et communicatif est construit sur l'apprentissage de séquences dont la structure temporelle est basée sur la répétition qui permet à l'enfant de maîtriser le temps par la régularité variée, la régularité ornée et diversifiée.

Presque toutes les conduites musicales ultérieures, dans toutes les cultures, sont basées sur ce principe : s'il paraît si universel, c'est bien qu'il est aussi profondément ancré dans la nature biologique de l'homme. Mais en même temps, le rythme des actions, des gestes, des mimiques, des vocalisations est un rythme partagé par les partenaires, et c'est ce partage, donc cette syntonie, cet "accordage" des états affectifs et moteurs qui permet la communication et l'échange. Ce que certains auteurs appellent la "musicalité " du comportement est bien en fait une capacité à réguler et harmoniser son propre comportement sur celui d'autrui pour communiquer sous des formes qui n'ont rien à voir avec le langage. Ainsi, dans les relations mèreenfant, la séquence de jeu à deux (système dyadique dynamique) est bien à la fois structurante du premier échange social et de la première expérience du temps : la 
forme primitive de la séquence musicale que j'ai décrite dans ma grammaire évolutive en porte d'ailleurs la trace comme base de toute expressivité musicale à venir. Dans la succession orientée à droite de l'appui initial, du colmatage et de l'accentuation finale, l'appui initial, l'appui-élan, l'appoggiatura au sens premier du terme (l'arsis aristotélicienne si l'on veut) est ouverture de soi vers l'extérieur, tension vers le partenaire, alors que le repos final qui clôt le processus de colmatage (thèsis) est fermeture, retour à soi, conclusion d'une séquence temporelle qui semble ainsi se constituer en unité désormais renvoyée au passé. Telle est selon moi la base à la fois psychologique et sociale des premiers schèmes d'ordre dans la production musicale des très jeunes enfants : comme toute forme de pensée et de représentation, ils s'enracinent dans le jeu des tensions et détentes de la tonicité et l'interaction de la tonicité individuelle avec la société qui, selon les cultures, vient la moduler, la contrôler et la codifier dans des systèmes linguistiques, musicaux ou symboliques. De ce point de vue, les échelles, les systèmes, les syntaxes sont seconds par rapport aux schèmes créateurs de structures temporelles, qu'elles soient musicales, sensorimotrices, ou affectives et sociales.

Mais voici l'idée la plus en prise avec tout ce que nous venons de voir : le contenu de la mémoire affective et sociale qui se constitue au cours des premiers mois de la vie est d'abord un contenu temporel, et c'est ce contenu temporel qui permet à l'enfant de comprendre très tôt tout ce qui relève de l'expressivité des schèmes temporels, qu'ils soient support de représentations sociales ou de figures musicales. En effet, lors de chaque interaction avec sa mère, l'enfant retient de ses jeux avec elle une forme particulière de l'alternance des répétitions et des variations, des états de tension, d'excitation et de détente qui en résultent. Cette forme est engrammée en mémoire de manière a-modale, globale, syncrétique. II n'en reste en somme qu'un contour temporel, un parcours qui se délimite dans l'expérience du sujet par un début et une fin. Pour chaque situation interactive, on imagine facilement que le même processus se déroule, ef très vite, l'enfant acquiert un stock très riche de contours temporels abstraits de séquences comportementales à deux qui sont ce que D. Stern appelle des "schémas-d'être-avec", être avec une personne dans telle et telle situation, dans tel contexte, dans telle action, dans tel jeu.

l'hypothèse est alors que l'unité d'une expérience interpersonnelle ou interactive est sa structure temporelle, sur laquelle se greffent les expériences sensorielles, motrices et affectives pour constituer des représentations intériorisées. Elle est en somme le prototype de toutes les expériences temporelles, par le fait qu'elle est une première "découpe", une première "segmentation" du temps dans le continuum des sensations désordonnées. II apparaît donc clairement que toute expérience et foute représentation d'expérience d'échange et de communication est une expérience vécue, dès les premiers instants de la socialisation, comme une entité temporelle. Tout acte, tout ressenti de cet acte, tout affect qui l'accompagne est du temps vécu, est une trame de temps. Les premiers schèmes d'ordre qui organisent les premières manifestations vocales de l'enfant sont issus de ces trames de 
temps engrangées dans l'expérience affective : d'où le concept d'affect de vitalité que développe Daniel Stern, pour qui les affects de vitalité sont des caractères des émotions qui sont d'abord de nature "dynamique". Ils sont donc liés aux émotions mais ne sont pas réductibles aux catégorisations abstraites, ils sont autant de nuances subtiles ressenties immédiatement et en même temps intraduisibles dans le vocabulaire référencié. Ils sont liés aux façons d'être, aux diverses façons de ressentir intérieurement les émotions. Ce sera, par exemple, tout ce qui sépare une joie "explosive" d'une joie "fugace», ou bien ce sera encore les mille façons de sourire, de se lever de sa chaise, de prendre le bébé dans ses bras, ressentis qui ne sont pas réductibles aux affects catégoriels classiques, mais qui viennent les colorer de manière toujours très sensible pour le sujet.

La comparaison avec la musique ou la danse s'impose de nouveau, car le chorégraphe ou le compositeur, eux aussi, traduisent bien plus une façon de sentir qu'un sentiment particulier : lorsque c'est le cas, ce qui fait l'originalité stylistique, c'est précisément que le sentiment abstrait traduit n'est jamais traduit de la même façon, c'est-à-dire ressenti de la même façon par deux compositeurs différents. Par exemple, la tristesse qu'évoque la musique de Debussy n'est pas la tristesse qu'évoque la musique de Brahms ou celle de Schumann, elles n'appartiennent pas au même univers de connotations affectives et sémantiques. Sans doute atteignonsnous là aussi la dimension stylistique : entre la tristesse et la joie debussyste, il y a une connivence particulière qui les différencie en même temps de la tristesse et de la joie de Brahms ou de Ravel. C'est bien ce que dit D. Stern : "Au cours des comportements spontanés, le domaine des affects de vitalité est l'équivalent du style dans l'art» (1985, trad. française, p. 206). Les affects de vitalité modulent, "stylisent » des programmes comportementaux fixes et rigides comme la marche, le sourire, etc. Tous les humains marchent, mais, alors que je ne distingue pas encore vos traits, au loin, je vous reconnais à votre démarche...

De plus, la notion correspondant à celle d'affect de vitalité en musique est sans doute celle qui caractérise les aspects dynamiques et temporels des formes musicales, il s'agit de la notion de vecteur dynamique. Les vecteurs dynamiques, parce qu'ils véhiculent des qualités vécues particulières du temps, véhiculent aussi des affects de vitalité, à moins que ce ne soient les affects de vitalité reconnus et éprouvés par le sujet qui soient projetés sur les vecteurs dynamiques en tant qu'organisateurs de la temporalité musicale. Par exemple, dans un travail antérieur (Imberty, 198 lb), j'avais demandé à des sujets musiciens et non musiciens, de décrire verbalement ce qu'ils entendaient, en temps réel au cours de l'audition d'une pièce de Debussy, La Puerta del Vino. Ainsi, à ce qu'on peut appeler la coda de la pièce, il y a un brusque passage à l'octave aiguë qui se fait pp. Les réponses indiquaient "sérénité, immobilisation, calme, extinction, effacement". Le passage à l'aigu a donc été ressenti dans ce ralentissement du temps (bien qu'il n'y ait pas de ralentissement objectif du tempo dans l'interprétation choisie), par cette "immobilisation ou cette sérénité " qui détend la densité de la durée et de 
l'écriture. Le passage à l'octave aiguë n'est donc pas seulement un changement de registre, mais prend la signification d'une sorte d'ouverture et d'immobilisation du temps musical, que le dernier arpège de la pièce vient subitement briser. $\mathrm{Ce}$ changement perçu et ressenti est donc un vecteur dynamique qui oriente la perception de l'auditeur, son attente, ses représentations internes. La qualité de cette orientation dépend de ce à quoi renvoie le vecteur dynamique, assimilé ici à un ensemble d'affects de vitalité dont l'auditeur fait immédiatement, à l'audition, l'expérience ou la reviviscence. Ce caractère "immédiat», «intuitif» de la compréhension des vecteurs dynamiques est la raison pour laquelle ils n'ont pas de réalité sémiologique objectivable. Is constituent seulement, dans la perception des sujets, les repères d'une intention de sens qui se dégage de la forme en devenir et du style qui la déborde de toutes parts.

Ainsi, cette orientation ressentie intimement, ou plutôt cette orientation temporelle toujours possible d'une expérience vécue intimement dans un geste vocal ou instrumental, ressentie comme lui étant cosubstantielle, et sans laquelle le sujet ne pourrait sans doute s'en approprier le sens, c'est ce qui constitue le ressort psychologique essentiel de toute la pensée musicale. Sans la prise en compte de cette dimension fondamentale de l'expérience musicale tant aux niveaux les plus élémentaires des débuts de la vie, qu'aux niveaux plus complexes de la production spontanée et de la créativité des enfants, qu'enfin au niveau de la création musicale elle-même, ni la psychologie ni la musicologie ne pourront rendre compte du temps comme substance de la forme musicale.

C'est sans doute là l'un des enjeux majeurs pour l'avenir de la recherche en psychologie de la musique.

\section{Références}

BEVER, T. et CHIARELLO, R. (1974), "Cerebral Dominance in Musicians and Non Musicians》, Science, 185, 537-539.

CHOMSKY, N. (1957) [1969], Structures syntaxiques. trad. française, Paris, Seuil.

CHOUVEL, J. M. (1998), La forme et le sens. Les métamorphoses d'Orphée, Paris, L'Harmattan, $180 \mathrm{p}$.

CUDDY, L. et BADERTSCHER, B. (1987), "Recovery of the Tonal Hierarchy : some Comparisons across Age and Levels of Musical Experience.", Perception and Psychophysics, 41, 609-620.

DELIĖGE, C. (1984), Les fondements de la musique tonale, Paris, J.C. Lattès, 271 p. FODOR, J. (1983), The Modularity of Mind, Cambridge, Mass., M.I.T. Press, 412 p.

GARDNER, H. (1983), Frames of Mind, the Theory of Multiple Intelligences, New York, Basic Books, 290 p. 
IMBERTY, M. (1969), L'acquisition des structures tonales chez l'enfant, Paris, Klincksieck, $226 \mathrm{p}$.

IMBERTY, M. (198 la), "Acculturation tonale et structuration perceptive du temps musical chez l'enfant», Basic Musical Functions and Musicals Ability, Stockholm, Publ. of Royal Swedish Academy of Music, 81-107.

IMBERTY, M. (1981), Les écritures du temps, Paris, Dunod, 274 p.

IMBERTY, M. (1982), "Può il concetto di grammatica esserci utile per l'elaborazione di una teoria della percezione musicale presso il bambino? ". Analisi musicale, computer, grammatica, Convegno internazionale, Modena, Pubbl. dans Rivista italiana di Musicologia, 1983, Firenze, Olschki, 255-271.

IMBERTY, M. (1991), "Le concept de hiérarchie perceptive face à la musique atonale» Communicazioni scientifiche di Psicologia Generale, Edizioni Scientifiche Italiane, 5, $119-133$.

JACKENDOFF, R. (1987), Consciousness and the Computational Mind, Cambridge, Mass., M.I.T. Press, $319 \mathrm{p}$.

LERDAHL, F. et JACKENDOFF, R. (1983), A Generative Theory of Tonal Music, Cambridge, (Mass), M.I.T. Press, 368 p.

MEYER, L. B. (1973) [1978], Explaining Music, seconde édition, Chicago University Press, $295 \mathrm{p}$.

MIALARET, J. P. (1997), Explorations musicales instrumentales chez le jeune enfant, Paris, Presses universitaires de France, $231 \mathrm{p}$.

MIROUDOT, L. (2000), La tonalité et la temporalité musicale chez l'enfant, texte introductif : IMBERTY, M., "Vers une psychologie des systèmes dynamiques", Paris, l'Harmattan, $235 \mathrm{p}$.

MOLINO, J. (1998), «Expérience et connaissance de la musique à l'âge des neurosciences", dans DARBELLAY, E., Le temps et la forme. Pour une épistémologie de la connaissance musicale. Genève, Droz, 253-272.

PERETZ, I. et MORAIS, J. (1989), "La musique et la modularité", dans MCADAMS, S. et DELIEGE, I., La musique et les sciences cognitives, Bruxelles, Mardaga, 393-414.

STERN, D. (1977), Mère-enfant, les premières relations, Bruxelles, Mardaga, 198 p.

STERN, D. (1985), Le monde interpersonnel du nourrisson, drad. française LAZARTIGUES, A. et CUPA-PERARD, D., Paris, Presses universitaires de France, $381 \mathrm{p}$.

TIBERGHIEN, G. (1999), "La psychologie cognitive survivrat-elle aux sciences cognitives? », Psychologie française , 44, 3, 265-283.

TREVARTHEN, C. (1999-2000), "Musicality and the Intrinsic Motive Pulse: Evidence from Human Psychobiology and Infant Communication », Musicæ Scientiæe, 155-215. 


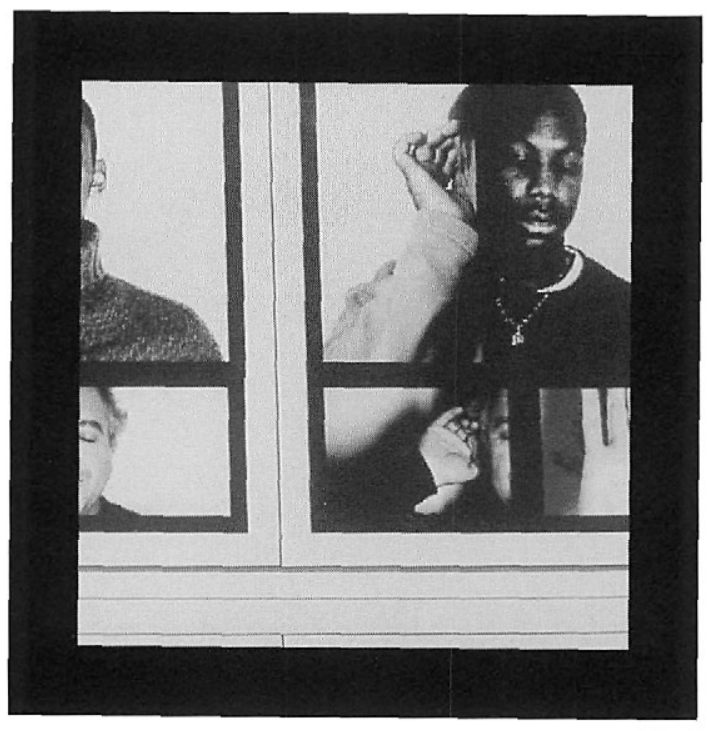

